\title{
Characterization of a-Si:H/c-Si Heterojunctions by Time Resolved Microwave Conductivity Technique
}

\author{
Amornrat Limmanee, ${ }^{1}$ Joaquim Nassar, ${ }^{2}$ Igor P. Sobkowicz, ${ }^{2,3}$ Jaran Sritharathikhun, \\ Kobsak Sriprapha, ${ }^{1}$ Guillaume Courtois, ${ }^{2,3}$ Francois Moreau, ${ }^{2}$ and Pere Roca I Cabarrocas ${ }^{2}$ \\ ${ }^{1}$ Solar Energy Technology Laboratory, National Electronics and Computer Technology Center, 112 Thailand Science Park, \\ Phahonyothin Road, Klong 1, Klong Luang, Pathumthani 12120, Thailand \\ ${ }^{2}$ LPICM, CNRS-Ecole Polytechnique, Route de Saclay, 91128 Palaiseau, France \\ ${ }^{3}$ Total New Energies, 24 Cours Michelet, La Défense 10, 92069 Paris La Défense, France
}

Correspondence should be addressed to Amornrat Limmanee; amornrat.limmanee@nectec.or.th

Received 17 December 2013; Revised 8 January 2014; Accepted 27 January 2014; Published 3 March 2014

Academic Editor: Peter Rupnowski

\begin{abstract}
Copyright (C) 2014 Amornrat Limmanee et al. This is an open access article distributed under the Creative Commons Attribution License, which permits unrestricted use, distribution, and reproduction in any medium, provided the original work is properly cited.

In heterojunction solar cells, a-Si:H/c-Si heterointerface is of significant importance, since the heterointerface characteristics directly affect junction properties and thus solar cell efficiency. In this study, we have performed time resolved microwave conductivity (TRMC) measurements on n-type c-Si wafers passivated on both sides with intrinsic and doped a-Si:H layers in order to investigate electrical property and passivation quality of the a-Si:H/c-Si heterojunctions. It was found that the TRMC decay time and decay curve shape varied with the laser wavelength and power intensity and also depended on sample structures. By using $1064 \mathrm{~nm}$ laser pulse with high excitation, differences in the decay curve shape between samples with and without p-n junction were observed. The samples containing p-n junction(s) had unique slow decay mode, after the initial fast decay, which we ascribed to the release of carriers from the low-mobility amorphous layer into the high-mobility crystalline wafer as the built-in field of the junction was restored. Experimental results suggest that the TRMC is useful nondestructive technique which is suitable for primary check of the a-Si:H/c-Si heterojunctions during the solar cell fabrication process.
\end{abstract}

\section{Introduction}

Heterojunction silicon solar cells, where hydrogenated amorphous silicon (a-Si:H) is deposited onto crystalline silicon (c$\mathrm{Si}$ ) wafers to provide both surface passivation and junction formation, receive great attention nowadays owing to known advantages over conventional Si wafer based solar cells, such as low temperature fabrication process, high efficiency, and superior performance at high operating temperature [1-3]. Monitoring of properties of such heterojunctions in the early stages of solar cell production is an essential concern. Microwave photoconductivity decay ( $\mu$-PCD) measurement techniques are routinely used to assess the surface passivation in a nondestructive, contactless way $[4,5]$. These techniques make use of the fact that photogenerated free carriers induce a change in the microwave reflectivity of a semiconductor material, the magnitude of which is governed by the mobility and excess density of the free carriers; the latter one is obtained from the photocarrier generation rate and the minority carrier effective lifetime $\left(\tau_{\text {eff }}\right)$. In high-quality crystalline silicon wafers, the $\tau_{\text {eff }}$ is dominated by surface rather than bulk recombination processes, making surface passivation a key issue.

Time resolved microwave conductivity (TRMC) experiment is a $\mu$-PCD setup, where illumination is achieved through a very short laser pulse that offers the possibility to investigate fast recombination mechanisms, such as those occurring in microcrystalline $\mathrm{Si}$ and organic materials $[6$, 7]. There have been several reports of investigations of hydrogenated amorphous silicon nitride $(\mathrm{a}-\mathrm{SiN}: \mathrm{H}) / \mathrm{c}-\mathrm{Si}$ and a-Si:H/c-Si heterojunctions by the TRMC technique [8-11]. However, they are mainly mentioned in case of an intrinsic $\mathrm{a}-\mathrm{Si}(\mathrm{N}): \mathrm{H} / \mathrm{c}-\mathrm{Si}$ heterojunctions and single side passivated samples. TRMC measurements on both sides of passivated 
samples and characterizations of doped a-Si:H/c-Si heterojunctions have been rarely reported [12]. In this study, we thus have performed TRMC measurements on two-side a$\mathrm{Si}: \mathrm{H}$ passivated $\mathrm{n}$-type c-Si substrates, focusing on changes in decay time and decay curve shape of different test structures. Kinetics which caused those variations were also analyzed.

\section{Experimental Details}

In these experiments, an excess of charge carriers was produced by illumination with $3 \mathrm{~ns}$ full width at half maximum (FWHM) pulses of a Nd:YAG laser at 532 and $1064 \mathrm{~nm}$. Optical filters were used to adjust incident light intensity over four orders of magnitude. The microwave probe signal was generated by a Gunn diode and directed via a circulator to the sample. The reflected microwave signal was detected from the sample via a circulator to a detector connected to a transient digitizer. $n$-doped, (100-) oriented, FZ grown silicon wafers of $280 \mu \mathrm{m}$-thickness and resistivity of $1-10 \Omega \mathrm{cm}$ were used as substrates. Two-side passivated samples with three different structures are made:

(a) $n$ a-Si:H/i a-Si:H/n c-Si/i a-Si:H/n a-Si:H (n-type a$\mathrm{Si}: \mathrm{H}$ on both sides),

(b) $p$ a-Si:H/i a-Si:H/n c-Si/i a-Si:H/p a-Si:H (p-type a$\mathrm{Si}: \mathrm{H}$ on both sides),

(c) $p$ a-Si:H/i a-Si:H/n c-Si/i a-Si:H/n a-Si:H (cell structure).

The intrinsic and doped a-Si:H films are prepared by radiofrequency plasma enhanced chemical vapor deposition (13.56 MHz rf-PECVD) technique. The gas sources are silane $\left(\mathrm{SiH}_{4}\right)$ and hydrogen $\left(\mathrm{H}_{2}\right)$. Phosphine $\left(\mathrm{PH}_{3}\right)$ and diborane $\left(\mathrm{B}_{2} \mathrm{H}_{6}\right)$ were used as dopant sources for $\mathrm{n}$-type and p-type, respectively. The thickness of the $i, n$, and p layers was about 8,28 , and $30 \mathrm{~nm}$, respectively. The test samples in this study were also measured with a SINTON lifetime tester (photoconductive decay technique) in the transient mode (short flash) for the comparison of decay time. It should be noted that attention was placed on trends among samples not the absolute value obtained from the measurements since the results varied with the measurement conditions of each technique.

\section{Results}

3.1. Power and Wavelength Dependent Behavior of TRMC Decay Curve. With increasing laser intensity the microwave reflectivity decay time of all test samples increased. And a significantly longer decay time was observed when the samples were stimulated by long laser wavelength, $1064 \mathrm{~nm}$, as shown in Figure 1. At high excitation an initial fast decay in the first $80-100 \mu$ s was observed. By measuring under exactly the same excitation conditions, it was found that TRMC decay time D.T. (sample a) > D.T. (sample c) > D.T. (sample b) (Figure 2). The sample with the $\mathrm{n}$ a-Si:H/i a-Si:H passivated on both sides (sample a) indicated the longest decay time while the sample with the $\mathrm{p}$ a-Si:H/i a-Si:H on both sides (sample b) showed the shortest decay time.

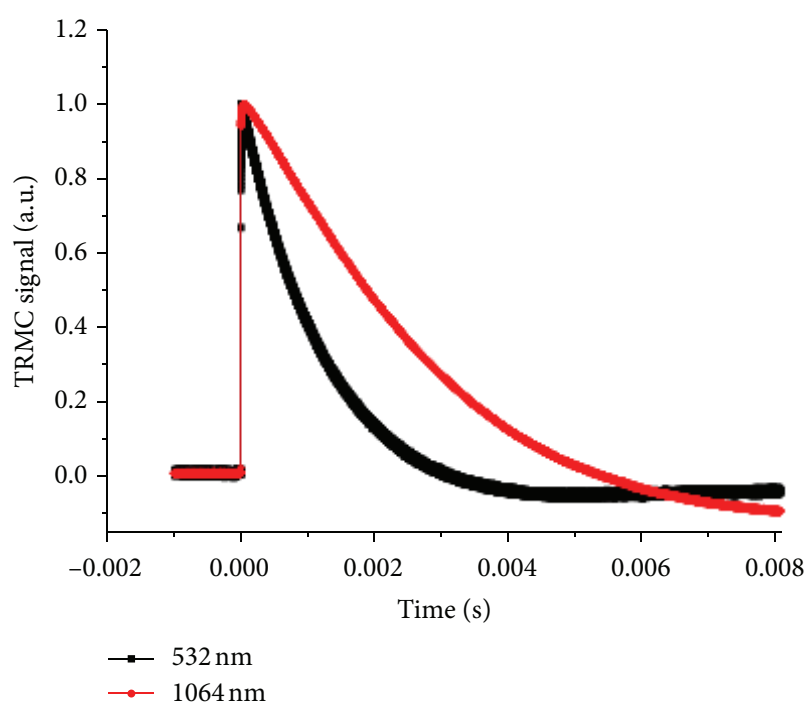

FIGURE 1: TRMC decay curves of sample with $\mathrm{n}$ a-Si:H on both sides induced by 532 and $1064 \mathrm{~nm}$.

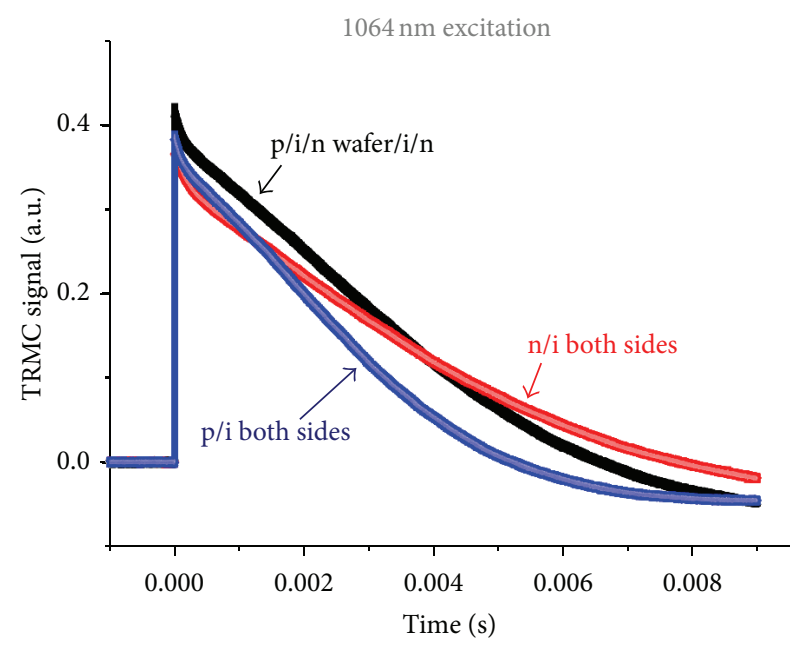

FIgURE 2: TRMC decay curves of samples with different structures; (a) both sides $\mathrm{n}$ a-Si:H, (b) both sides p a-Si:H, and (c) cell structure, under the same illuminated conditions.

The $\tau_{\text {eff }}$ of the samples with different structures estimated by using the SINTON lifetime tester is summarized in Table 1. It can be seen that the $\tau_{\text {eff }}$ and the decay time from the TRMC measurements of these three structures exhibit the same trend; that is to say, $\tau_{\text {eff }}(\mathrm{a})>\tau_{\text {eff }}(\mathrm{c})>\tau_{\text {eff }}$ (b). The effective lifetime of various samples from the TRMC measurements was summarized as a function of number of photon of laser pulse in Figure 3. Here, the decay curves were deemed to be exponential decay and an exponential fit yielded a 1/e lifetime as the $\tau_{\text {eff }}$. As the number of photon increased (higher light intensity), the decay time of all samples increased and tended to become saturated at the decay time of about $4 \mathrm{~ms}$, which was likely to be a bulk lifetime of the n-type crystalline wafer used in this study. 


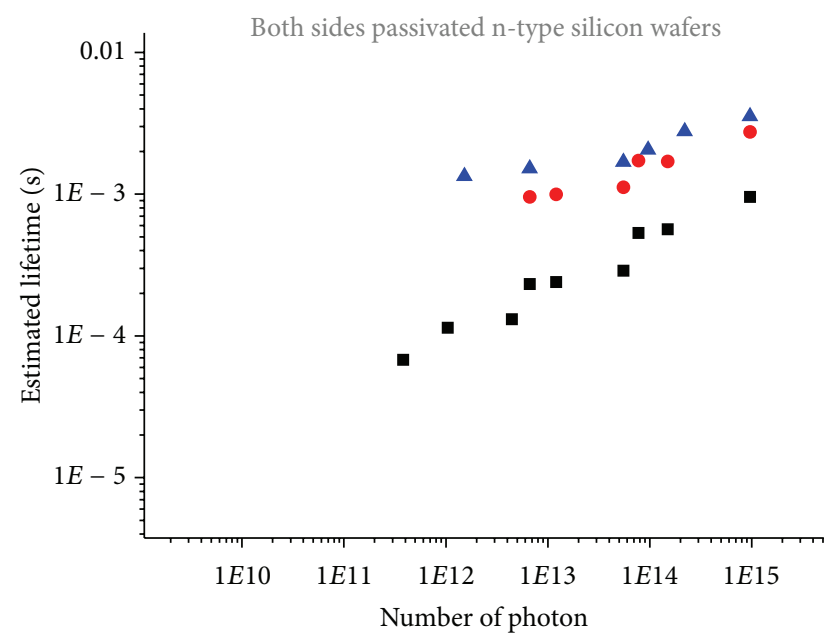

FIGURE 3: Estimated effective lifetime of various samples as a function of number of photon.

3.2. Structure Dependent Behavior of TRMC Decay Curve. By using $1064 \mathrm{~nm}$ excitation, differences in the decay curve shape between samples with and without $\mathrm{p}-\mathrm{n}$ junction were found. The decay curve shape of the samples with $\mathrm{p}-\mathrm{n}$ junction, samples b and c, can be divided into three portions: (1) initial fast decay mode, (2) slow decay mode, and (3) principal decay mode, as exhibited in Figure 4. The samples without $\mathrm{p}-\mathrm{n}$ junction seemed to contain only portions (1) and (3).

\section{Discussions}

The differences in the TRMC signal-both decay time and curve shape-implied the variations in the passivation quality and the junction properties. Analysis of these variations could lead to better understanding of recombination kinetics of the heterojunctions. It is worth noting that two phenomena can contribute to a decrease of the TRMC signal in our heterojunction structures.

(i) The Electron-Hole Recombination. It is known that in amorphous region the bulk minority carrier lifetime is less than a few $10^{-8} \mathrm{~s}$, while in high-quality silicon it is in the millisecond range.

(ii) The Transfer of Carriers from a Crystalline to an Amorphous Region. Indeed, their mobility in the latter is three orders of magnitude below their mobility in crystalline silicon [11].

This allows us to qualitatively understand the shape of the response curves obtained for the structures with $\mathrm{p}$ a-Si:H/n cSi on one or two sides (Figure 2).

(i) As the built-in electric field keeps the free carrier density to a very low level at the location of the heterojunction, holes generated in the high-mobility n-type silicon crystal diffuse towards the low-mobility p-type amorphous silicon region. Once they enter it, their contribution to the TRMC signal is expected to drop sharply. Yet the resulting charge separation

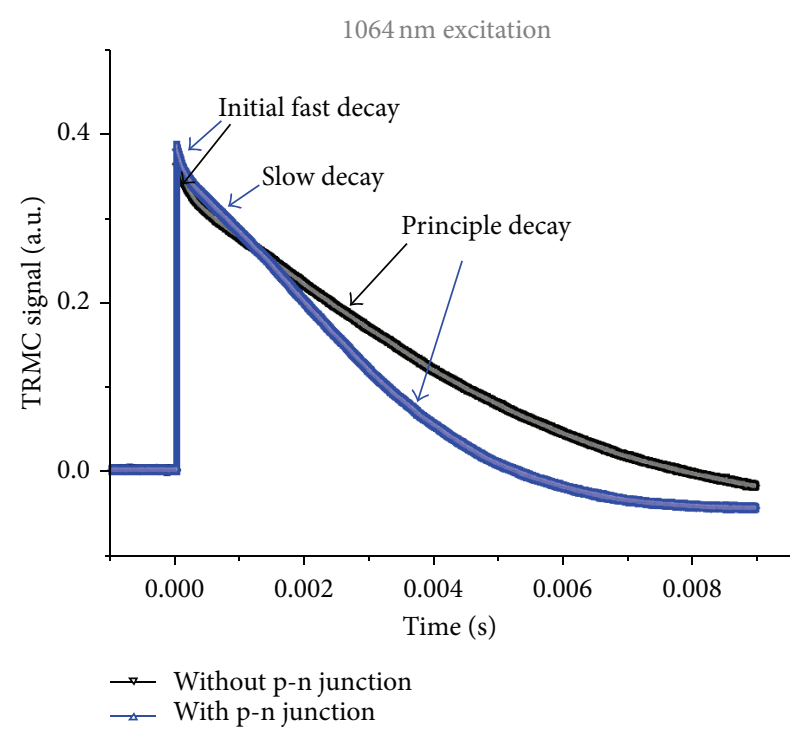

FIGURE 4: Comparison of TRMC decay curves between samples with and without $\mathrm{p}-\mathrm{n}$ junction.

induces a growing counter electric field that ends up blocking further diffusion. The timescale of the transient diffusion regime can be estimated to be $w^{2} / D_{p}$ where $w$ is the thickness of the wafer (about $300 \mu \mathrm{m})$ and $D_{p}$ is the diffusion constant for minority holes in $n$-doped crystalline silicon (about $10 \mathrm{~cm}^{2} \cdot \mathrm{s}^{-1}$ at room temperature). Accordingly, we obtained a value of $90 \mu \mathrm{s}$, which corresponded to the typical timescale of the observed initial fast decay (Figure 4).

(ii) As recombination goes on in the crystalline silicon region, holes that were stored in the amorphous region are released back into the crystalline regiongradually bringing the electric field back to the built-in equilibrium value of the heterojunction. The decrease of the TRMC response due to recombination is therefore slowed down by the reverse transfer of holes from a low-mobility region back to a highmobility region, leading to the "slow decay" part of the TRMC response in Figure 4.

(iii) After the electric field recovers its initial value then the "principle mode" sets in. The principle mode, exponential decay portion, can be observed at any illumination intensity.

Among the three structures, the sample with n-type aSi:H passivated on both sides indicates the longest decay time. The front and rear sides of this structure act as reflecting walls for minority carriers, having excellent passivation property and preventing transfer of holes to the low-mobility layers [11]. Its decay kinetic can be simply expressed in the form of one exponential function. The "slow decay" mode is not observed. Longest lifetime is observed when the excitation wavelength is $1064 \mathrm{~nm}$, corresponding to excess charge carrier generation in the bulk of the $\mathrm{c}$-Si wafer and negligible absorption in the a-Si:H layer, as opposed to an excitation 
TABLE 1: Effective lifetime $\left(\tau_{\text {eff }}\right)$ of samples with various structures measured by SINTON lifetime tester in the transient mode at minority carrier density of $2 \times 10^{15} \mathrm{~cm}^{-3}$.

\begin{tabular}{lcc}
\hline Sample & Structure & Effective lifetime $(\mathrm{ms})$ \\
\hline $\mathrm{a}$ & $\mathrm{n} \mathrm{a}-\mathrm{Si}: \mathrm{H} / \mathrm{i}$ a-Si:H on both sides & 4.2 \\
$\mathrm{~b}$ & $\mathrm{p} \mathrm{a-Si:H/i} \mathrm{a-Si:H} \mathrm{on} \mathrm{both} \mathrm{sides}$ & 1.5 \\
$\mathrm{c}$ & Cell structure & 2.1 \\
\hline
\end{tabular}

wavelength of $532 \mathrm{~nm}$, which leads to initial excess charge carrier profile generation at the illuminated surface.

It is obvious that the TRMC results can provide information regarding not only the $\tau_{\text {eff }}$ but also the heterojunction properties, both of which are significantly important properties for the heterojunction silicon solar cell development. These demonstrate that the TRMC technique has high potential as an effective analysis tool for heterojunction silicon solar cells.

\section{Conclusions}

Based on information from the TRMC decay curves, the electrical property and passivation quality of the a-Si:H/cSi heterojunctions as well as their changes can be evaluated. The decay curve time and the decay curve shape of samples provided meaningful interpretation of excess charge carrier kinetics in the heterojunctions. The samples with $\mathrm{p}$ a-Si:H/n c-Si heterojunction (s) containing a depletion/inversion layer at the interfaces showed the unique slow decay mode at high excitation (after the initial fast decay mode) due to excess charge carrier stored in the amorphous regions. These results agreed well with the change in the $\tau_{\text {eff }}$ from the SINTON lifetime tester. Experimental results suggest that the TRMC is an useful nondestructive technique which is suitable for primary check of the thin film a$\mathrm{Si}: \mathrm{H} / \mathrm{c}-\mathrm{Si}$ heterojunctions during the solar cell fabrication process.

\section{Conflict of Interests}

The authors declare that there is no conflict of interests regarding the publication of this paper.

\section{Acknowledgment}

The authors gratefully acknowledge the support of the French Embassy in Thailand through the postdoctoral fellowship program for giving the opportunity to carry out this study.

\section{References}

[1] T. Mikio, K. Kunihiro, T. Sadaji et al., "HIT ells-high-efficiency crystalline Si cells with novel structure," Progress in Photovoltaics Research and Applications, vol. 8, pp. 503-513, 2000.

[2] M. R. Page, E. Iwaniczko, Y.-Q. Xu et al., "Amorphous/ crystalline silicon heterojunction solar cells with varying i-layer thickness," Thin Solid Films, vol. 519, no. 14, pp. 4527-4530, 2011.
[3] D. L. Batzner, Y. Andrault, L. Andreetta et al., "Properties of high efficiency silicon heterojunction cells," Energy Procedia, vol. 8, pp. 153-159, 2011.

[4] A. W. Stephens, A. G. Aberle, and M. A. Green, "Surface recombination velocity measurements at the silicon-silicon dioxide interface by microwave-detected photoconductance decay," Journal of Applied Physics, vol. 76, no. 1, pp. 363-370, 1994.

[5] N. Schüler, T. Hahn, K. Dornich, J. R. Niklas, and B. GründigWendrock, "Theoretical and experimental comparison of contactless lifetime measurement methods for thick silicon samples," Solar Energy Materials and Solar Cells, vol. 94, no. 6, pp. 1076-1080, 2010.

[6] S. Kasouit, J. Damon-Lacoste, R. Vanderhaghen, and P. Roca i Cabarrocas, "Contribution of plasma generated nanocrystals to the growth of microcrystalline silicon thin films," Journal of Non-Crystalline Solids, vol. 338-340, no. 1, pp. 86-90, 2004.

[7] S. Kasouit, P. Rca i Cabarrocas, and R. Vanderhaghen, "DTRMC, a probe of transverse transport in microcrystalline silicon," Thin Solid Films, vol. 427, no. 1-2, pp. 335-339, 2003.

[8] M. Kunst, G. Müller, R. Schmidt, and H. Wetzel, "Surface and volume decay processes in semiconductors studied by contactless transient photoconductivity measurements," Applied Physics A Solids and Surfaces, vol. 46, no. 2, pp. 77-85, 1988.

[9] S. von Aichberger, H. Feist, J. Löffler, and M. Kunst, "In situ monitoring of the deposition of a-Si:H/c-Si heterojunctions by transient photoconductivity measurements," Solar Energy Materials and Solar Cells, vol. 65, no. 1, pp. 417-422, 2001.

[10] M. Kunst, O. Abdallah, and F. Wünsch, "Passivation of silicon by silicon nitride films," Solar Energy Materials and Solar Cells, vol. 72, no. 1-4, pp. 335-341, 2002.

[11] M. Kunst, S. von Aichberger, G. Citarella, and F. Wünsch, "Amorphous silicon/crystalline silicon heterojunctions for solar cells," Journal of Non-Crystalline Solids, vol. 299-302, no. 2, pp. 1198-1202, 2002.

[12] F. Wünsch, G. Citarella, O. Abdallah, and M. Kunst, "An inverted a-Si:H/c-Si hetero-junction for solar energy conversion," Journal of Non-Crystalline Solids, vol. 352, pp. 1962-1966, 2006 

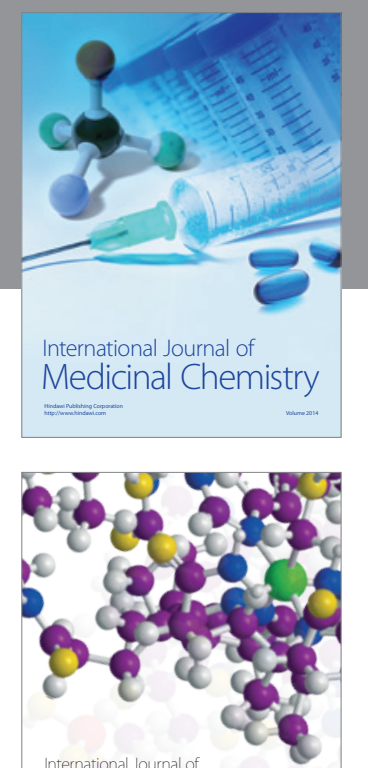

\section{Carbohydrate} Chemistry

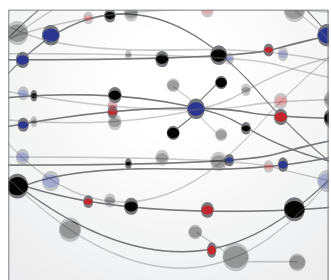

The Scientific World Journal
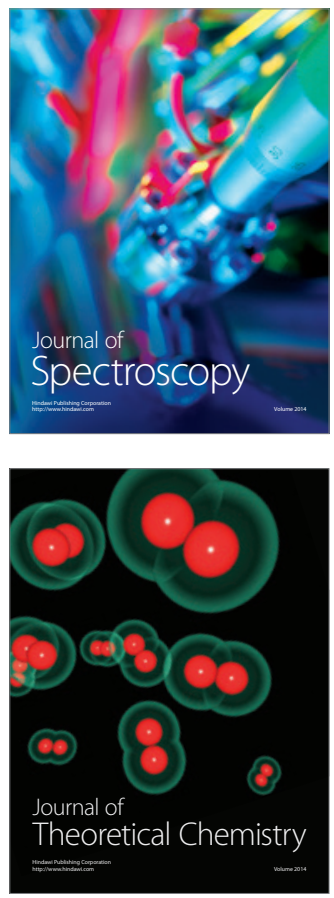
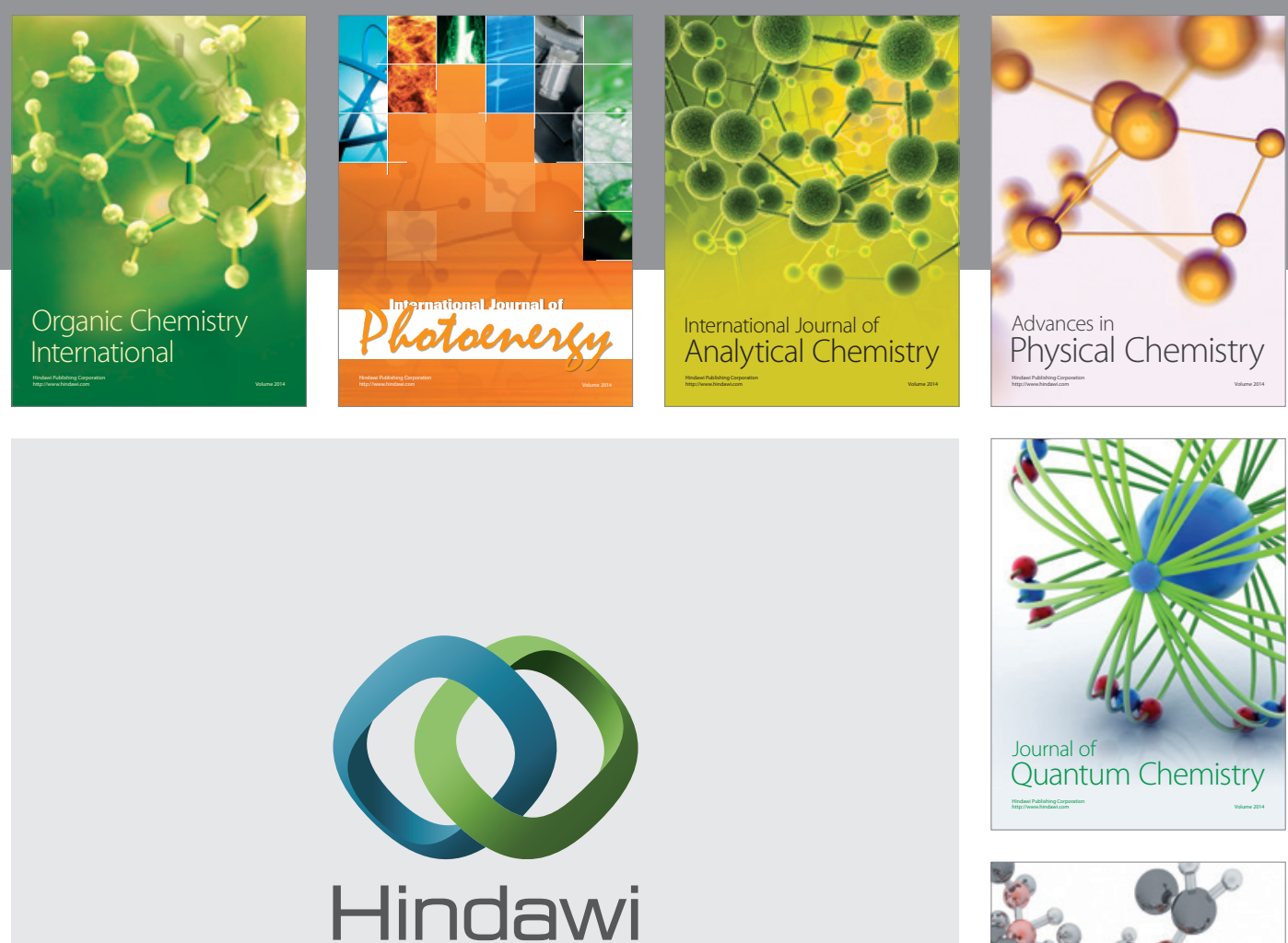

Submit your manuscripts at

http://www.hindawi.com

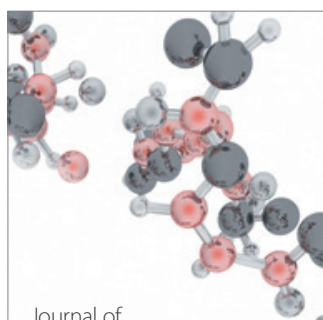

Analytical Methods

in Chemistry

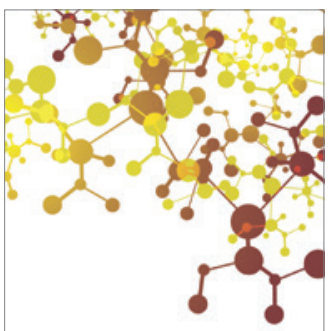

Journal of

Applied Chemistry

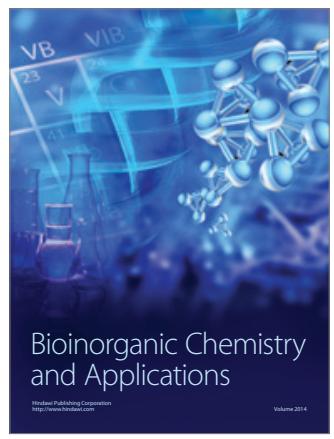

Inorganic Chemistry
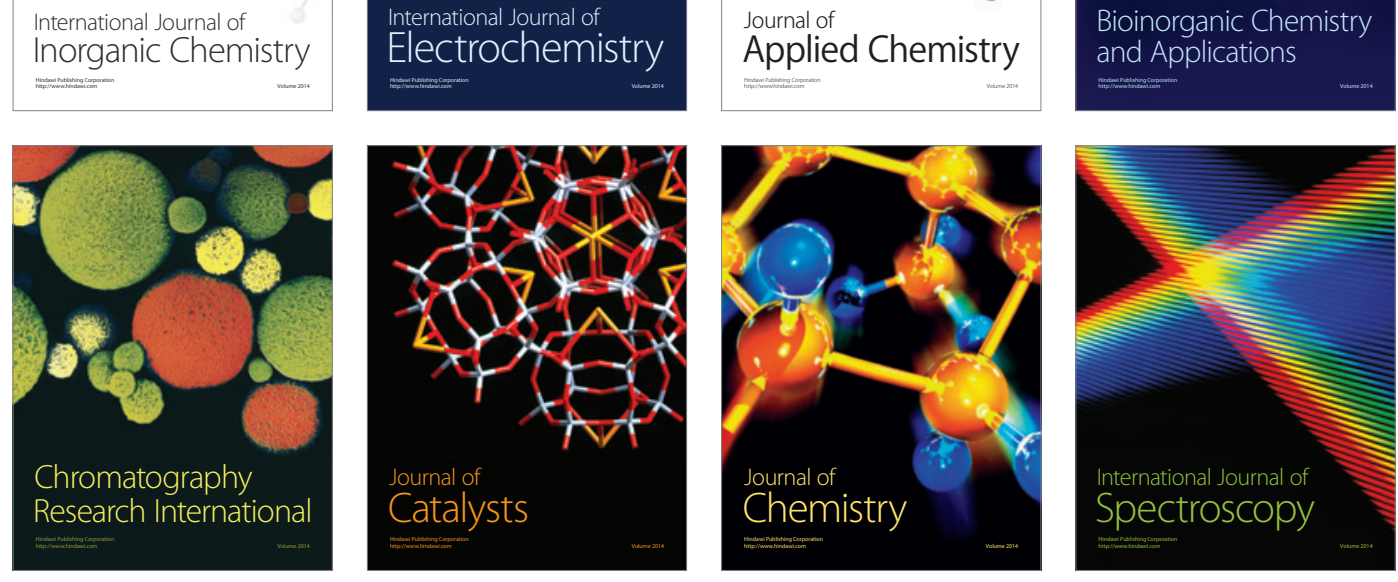